\title{
Experimental observation of band inversion in the PbSnTe system
}

\author{
S. O. Ferreira ${ }^{a)}$ \\ Departamento de Física, Universidade Federal de Viçosa, Viçosa, MG, Brasil \\ E. Abramof, P. Motisuke, P. H. O. Rappl, H. Closs, A. Y. Ueta, C. Boschetti, \\ and I. N. Bandeira \\ Lab. Associado de Sensores e Materiais, Instituto Nacional de Pesquisas Espaciais, São José dos Campos, \\ SP, Brasil
}

(Received 13 April 1999; accepted for publication 14 September 1999)

\begin{abstract}
The band inversion in the PbSnTe compound has been observed by optical and electrical measurements. The samples, high quality $\mathrm{Pb}_{1-x} \mathrm{Sn}_{x} \mathrm{Te}$ epitaxial layers, have been grown by molecular-beam epitaxy on $(111) \mathrm{BaF}_{2}$ substrates. Optical transmission measurements have revealed a change in signal of the energy gap temperature derivative for samples with $0.35<x<0.70$. In the same samples and at the same temperature, a minimum in the resistivity has been observed, showing a interrelation between the optical and electrical measurements. However, the temperature, for which the inversion occurs, is not that predicted by the band inversion model. This discrepancy is supposed to be due to the relatively high hole concentration of these samples. (C) 1999 American Institute of Physics. [S0021-8979(99)08624-7]
\end{abstract}

The lead-tin telluride alloy system has been investigated for many years and applied mainly in the fabrication of infrared photodetectors and diode lasers. ${ }^{1-3}$ New interesting research possibilities were opened by alloying with rareearth and magnetic elements such as $\mathrm{Eu}, \mathrm{Yb}$, and Mn. ${ }^{4,5}$ The growth of lead salts on silicon to obtain monolithic integration of detector arrays with silicon read-out circuits, has recently received much attention. ${ }^{6,7}$

According to the band inversion (BI) model, ${ }^{8}$ the $\mathrm{Pb}_{1-x} \mathrm{Sn}_{x}$ Te energy gap $\left(E_{g}\right)$ initially decreases as the $\mathrm{Sn}$ composition increases, and vanishes for an intermediate alloy composition. Further increasing the Sn composition, the $E_{g}$ starts to increase, with the band edge states inverted, up to the SnTe value. The $\mathrm{Sn}$ composition for which the band inversion should occur varies from $x \sim 0.35$ to $x \sim 0.65$ as the temperature increases from 4 to $300 \mathrm{~K}$. However, it is very difficult to determine the band edge structure near and beyond the band inversion region, since only high carrier concentration samples can be obtained. ${ }^{9}$ The energy gap of $\mathrm{Pb}_{1-x} \mathrm{Sn}_{x}$ Te has been experimentally determined only for $x$ $<0.25$. The Burstein-Moss shift, caused by the high hole concentration, which is observed in the samples with higher tin composition, imposes difficulties in the determination of the "real" gap by optical-absorption measurements. ${ }^{10,11}$ For higher $x$, the only $E_{g}$ experimental data available has been determined by tunneling measurements in $\mathrm{Al} / \mathrm{Al}_{2} \mathrm{O}_{3} / \mathrm{SnTe}$ structures. $^{12}$ The real $E_{g}$ of pure $\operatorname{SnTe}\left(p \sim 10^{20} \mathrm{~cm}^{-3}\right)$ is $0.18 \mathrm{eV}$, while the "optical energy gap" ( $\left.E_{g}^{\mathrm{opt}}\right)$ was found to be near $0.5 \mathrm{eV} .^{10,11}$

Using In doping to reduce the carrier concentration, Takaoka et al. ${ }^{13}$ have determined the effective masses and $E_{g}$ as a function of $x$, across the band-inversion region by the far-infrared magnetoplasma method. They have observed

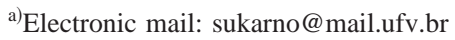

much heavier effective masses in these doped samples as compared to the values expected for undoped $\mathrm{Pb}_{1-x} \mathrm{Sn}_{x} \mathrm{Te}$ and an energy gap which does not go to zero, in contrary to the BI model. They concluded that it would be difficult to say if this behavior is essential for $\mathrm{PbSnTe}$ or just a result of In doping. Therefore, although the BI model is widely accepted, its direct observation, for the $\mathrm{Pb}_{1-x} \mathrm{Sn}_{x} \mathrm{Te}$ system, has not been achieved yet.

Recently, we have reported on the growth of high quality PbSnTe samples, covering the whole composition range. ${ }^{14}$ The layers were grown on (111) $\mathrm{BaF}_{2}$ substrates by molecular-beam epitaxy using solid PbTe and SnTe sources, at temperatures between 250 and $300^{\circ} \mathrm{C}$. The samples were characterized by high-resolution $\mathrm{x}$-ray diffraction, temperature-dependent resistivity, Hall mobility, and infrared transmission. The films, grown using stoichiometric $\mathrm{PbTe}$ and $\mathrm{SnTe}$ sources, have shown a hole concentration between $p=1 \times 10^{17} \mathrm{~cm}^{-3}$, for $\mathrm{Pb}_{0.85} \mathrm{Sn}_{0.15} \mathrm{Te}$, and $p=2$ $\times 10^{19} \mathrm{~cm}^{-3}$, for SnTe. The value, observed for $\mathrm{SnTe}$, is at least one order of magnitude lower than the one previously reported in the literature. Details about the electrical characterization of these layers have been published previously. ${ }^{15}$

In this work, we focus attention on the optical characterization of these layers, measuring the temperature dependence of the energy gap. $E_{g}$ has been determined from the transmission spectra, measured using a Fourier-transform infrared spectrometer in the range from 4500 to $800 \mathrm{~cm}^{-1}$, with a resolution of $2 \mathrm{~cm}^{-1}$. A liquid He cryostat was used to cool down the samples between 5 and $300 \mathrm{~K}$. At each desired temperature, the transmission spectra of a clean $\mathrm{BaF}_{2}$ substrate, taken just before the sample, was used as a background reference. Figure 1 shows $E_{g}$ as a function of temperature for two samples outside the BI region. As expected, the energy gap increases with temperature, for the sample with $x=0.15$, while it decreases, for the sample with $x$ $=0.82$. But, in contrast to the model, the absolute values of 


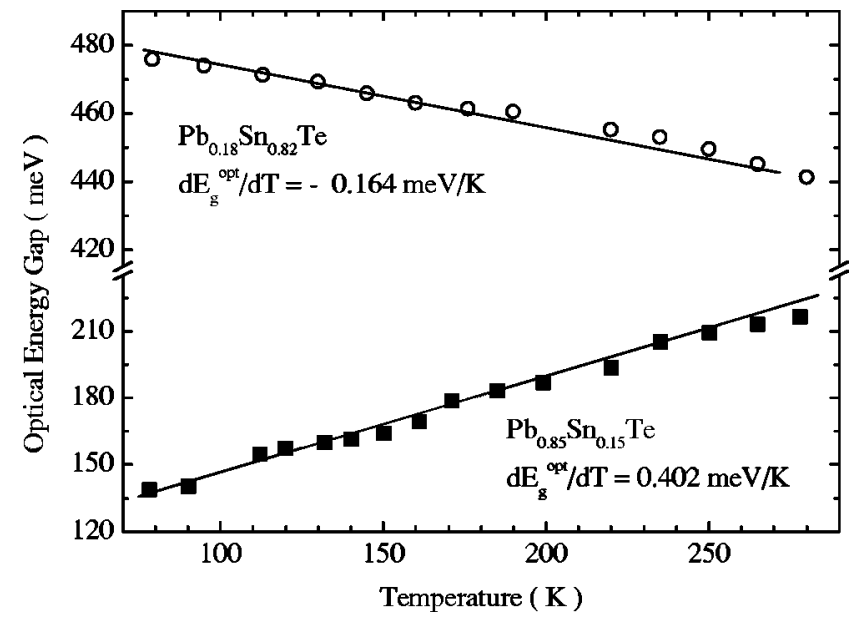

FIG. 1. Optical energy gap as a function of temperature for two $\mathrm{Pb}_{1-x} \mathrm{Sn}_{x} \mathrm{Te}$ samples outside the band inversion region. The solid lines are best linear fit to the data.

the energy gap temperature coefficients $\left(d E_{g} / d T\right)$ were very different. This occurs because the energy gap taken from the transmission spectra is $E_{g}^{\text {opt }}$, which takes in account the effect of the band filling, Burstein-Moss (BM) shift. For $\mathrm{Pb}_{0.85} \mathrm{Sn}_{0.15} \mathrm{Te}\left(p=3 \times 10^{17} \mathrm{~cm}^{-3}\right)$, the $\mathrm{BM}$ shift is negligible and the measured values of $E_{g}$ and $d E_{g} / d T$ are the expected ones. ${ }^{16}$ On the other hand, for the sample with $x=0.82$ ( $p$ $\left.=1 \times 10^{19} \mathrm{~cm}^{-3}\right), E_{g}^{\mathrm{opt}}$ is higher than $E_{g}$ by almost $230 \mathrm{meV}$ and the optical energy gap temperature coefficient $\left(d E_{g}^{\mathrm{opt}} / d T\right)$ is almost three times lower. The observed temperature dependence is a combination of the change in $E_{g}$ and in the position of Fermi level with temperature. Preliminary calculations of the absorption coefficient, using the formulas proposed by Anderson, ${ }^{17}$ which include the BM shift, can reproduce this decrease in $d E_{g}^{\mathrm{opt}} / d t$ for PbTe samples with high hole concentration, showing that the band filing is the responsible for this behavior. The results of these calculations will be published elsewhere.

The behavior of $E_{g}^{\mathrm{opt}}$ is completely different for the samples with $x$ between 0.35 and 0.7 , for which the band crossing should occur according to the BI model. Figure 2

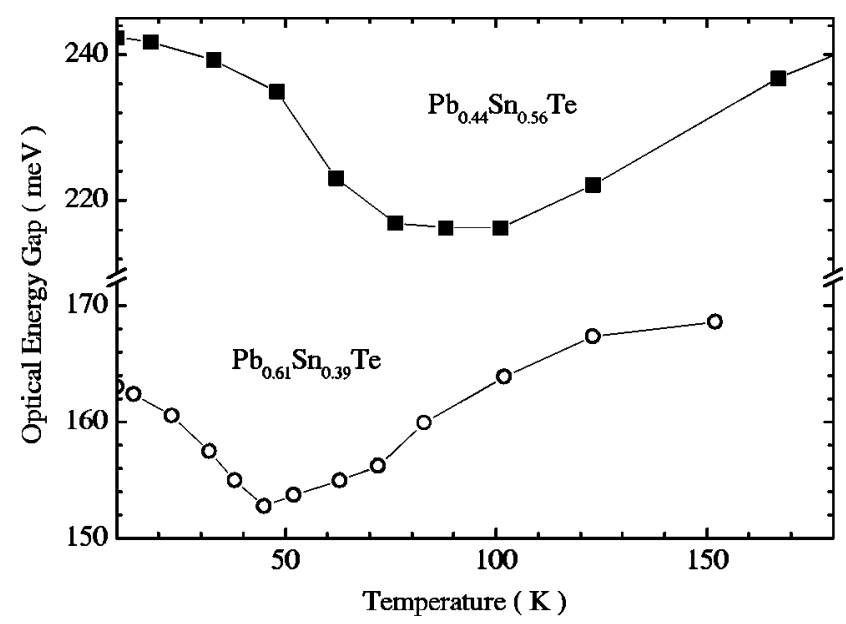

FIG. 2. Optical energy gap as a function of temperature for two $\mathrm{Pb}_{1-x} \mathrm{Sn}_{x} \mathrm{Te}$ samples in the band inversion region. The lines are guides to the eye.

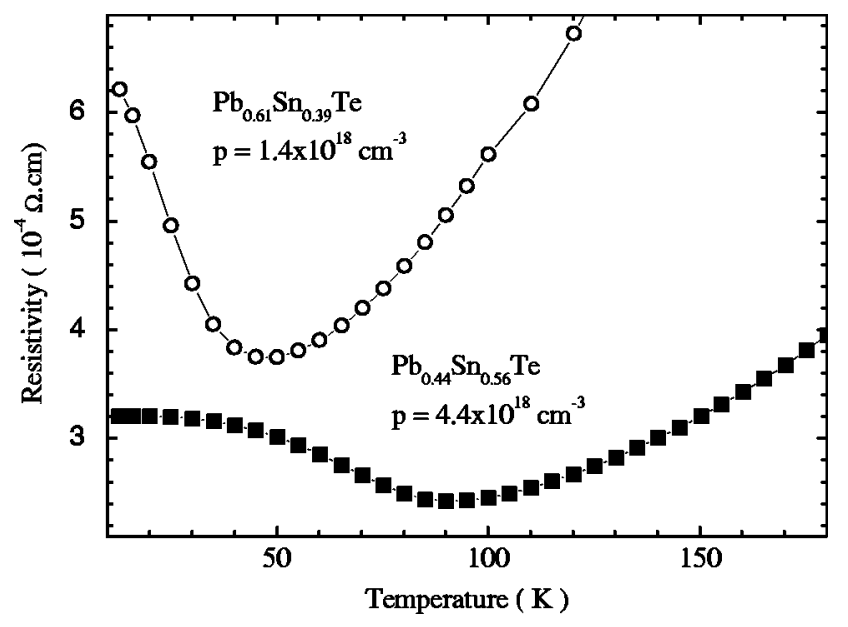

FIG. 3. Temperature dependence of resistivity for the same $\mathrm{Pb}_{1-x} \mathrm{Sn}_{x} \mathrm{Te}$ samples which are shown in Fig. 2.

shows the temperature dependence of the energy gap for two $\mathrm{Pb}_{1-x} \mathrm{Sn}_{x}$ Te samples with $x=0.39$ and $x=0.56$. In contrast to Fig. $1, E_{g}^{\text {opt }}$ first reduces, reaches a minimum and than starts to increase. Again, due to the relatively high carrier concentration of the samples (above $10^{18} \mathrm{~cm}^{-3}$ ), the temperature coefficient of $E_{g}^{\mathrm{opt}}$ is much smaller than the expected value. The change in signal of $d E_{g}^{\mathrm{opt}} / d T$, is a direct evidence of the band inversion in this alloy system.

The electrical characterization of these PbSnTe layers has also revealed two distinct behaviors, for samples, inside and outside the band inversion region. For $\mathrm{Pb}_{1-x} \mathrm{Sn}_{x}$ Te layers with $x$ outside the BI region, the resistivity always increases as a function of temperature. On the other hand, all samples with Sn composition in the BI region exhibit a welldefined minimum in the resistivity curve and a corresponding maximum in the mobility. Figure 3 shows the resistivity as a function of temperature for two samples in the BI region (the same shown in Fig. 2). This anomalous behavior is supposed to be related to the band crossing since it happens only for samples in the band inversion region.

Besides that, the temperature where the minimum in resistivity occurs correlates perfectly with the temperature of minimum $E_{g}^{\mathrm{opt}}$. This coincidence can be clearly seen in Fig. 4 , where we plot the temperature of minimum resistivity and minimum $E_{g}$ as a function of $\mathrm{Sn}$ content for all samples measured. The band crossing temperature expected from the BI model is also plotted in this figure. It can be observed that the experimental data are close to values predicted by the model only for samples with low tin composition and the difference between theory and experiment increases as a function of tin composition. This discrepancy, observed between the model and the experimental values, is not yet clearly understood, but we believe that the increasing carrier concentration in the $\mathrm{Pb}_{1-x} \mathrm{Sn}_{x}$ Te system with $x$, is responsible for this effect. As we have already mentioned, the high carrier concentration of the $\mathrm{PbSnTe}$ samples produces a strong Burstein-Moss shift, leading to a big change in the temperature dependence and absolute value of the $E_{g}^{\text {opt }}$. Since the BI model does not take this effect in account, we 


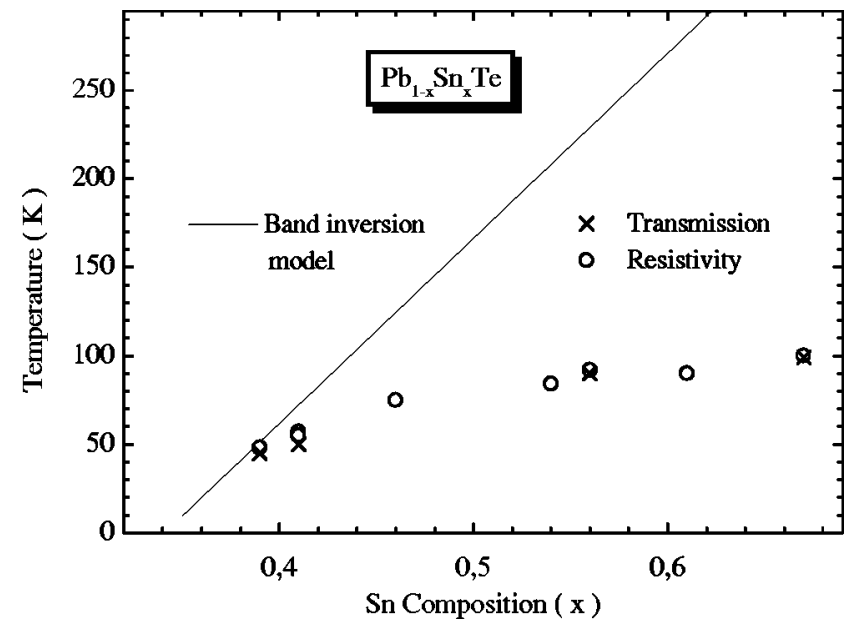

FIG. 4. Temperature of minimum resistivity $(\bigcirc)$ and of $d E_{g}^{\mathrm{opt}} / d T=0$ $(\times)$ as a function of tin composition $(x)$. The solid line is the band crossing temperature calculated from the band inversion model.

believe that this is probably the reason for the observed difference.

We have observed the change in signal of $d E_{g}^{\text {opt }} / d T$ for $\mathrm{Pb}_{1-x} \mathrm{Sn}_{x} \mathrm{Te}$ samples with $0.35<x<0.70$. This is the first direct experimental observation of the band inversion in this alloy. Besides that, we have shown that the band inversion can also be observed by means of electrical measurements, through the minimum in the resistivity as a function of temperature. The Burstein-Moss shift, which appears in the $\mathrm{PbSnTe}$ system due to the high hole concentration, produces a big change in the temperature dependence and absolute value of $E_{g}^{\mathrm{opt}}$. This effect is probably the reason for the dis- crepancy between the inversion temperature values predicted by the BI model and our experimental data.

This work has been partially supported by "Conselho Nacional de Desenvolvimento Científico e TecnológicoCNPq" and "Fundação de Amparo à Pesquisa do Estado de São Paulo-FAPESP."

${ }^{1}$ H. Holloway and J. N. Walpole, Prog. Cryst. Growth Charact. 2, 49 (1979)

${ }^{2}$ H. Preier, Appl. Phys. 20, 189 (1979).

${ }^{3}$ Y. Horikoshi, in Semiconductors and Semimetals, Vol. 22, Part C, edited by W. T. Tsang (Academic, New York, 1985), p. 93.

${ }^{4}$ D. L. Partin, in Semiconductors and Semimetals, Vol. 33, edited by T. P. Pearsall (Academic, New York, 1991), p. 311.

${ }^{5}$ G. Springholz and G. Bauer, J. Cryst. Growth 144, 157 (1994).

${ }^{6}$ C. Boschetti, P. H. O. Rappl, A. Y. Ueta, and I. N. Bandeira, Infrared Phys. 34, 281 (1993).

${ }^{7}$ H. Zogg, A. Fach, C. Maissen, J. Masek, and S. Blunier, Opt. Eng. (Bellingham) 33, 1440 (1994).

${ }^{8}$ J. Dimmock, I. Melngailis, and A. J. Strauss, Phys. Rev. Lett. 16, 1193 (1966).

${ }^{9}$ T. C. Harman, J. Nonmet. 1, 183 (1973).

${ }^{10}$ E. G. Bylander, J. R. Dixon, H. R. Riedl, and R. B. Schoolar, Phys. Rev. 138, A873 (1965).

${ }^{11}$ R. B. Schoolar, H. R. Riedl, and J. R. Dixon, Solid State Commun. 4, 423 (1966).

${ }^{12}$ L. Esaki and P. J. Stiles, Phys. Rev. Lett. 16, 1108 (1966)

${ }^{13}$ S. Takaoka, S. Shimomura, H. Takahashi, and K. Murase, Phys. Rev. B 40, 5642 (1989).

${ }^{14}$ P. H. O. Rappl, H. Closs, S. O. Ferreira, E. Abramof, C. Boschetti, P. Motisuke, A. Y. Ueta, and I. N. Bandeira, J. Cryst. Growth 191, 466 (1998).

${ }^{15}$ E. Abramof, S. O. Ferreira, P. H. O. Rappl, H. Closs, and I. N. Bandeira, J. Appl. Phys. 82, 2405 (1997)

${ }^{16}$ G. Nimtz, Numerical Data and Functional Relationships in Science and Technology, in: K. Hellwege, L. B. Madelung (eds.), New Series Group III, Vol. 17, Part F (Springer, Berlin, 1993), p. 168.

${ }^{17}$ W. W. Anderson, Infrared Phys. 20, 363 (1980). 